\title{
Research of a third-order nonlinear differential equation in the vicinity of a moving singular point for a complex plane
}

\author{
Victor Orlov ${ }^{*}$ and Magomedyusuf Gasanov ${ }^{\dagger}$ \\ Moscow State University of Civil Engineering, Yaroslavskoe shosse, 26, Moscow, 129337, Russia
}

\begin{abstract}
This article generalizes the previously obtained results of existence and uniqueness theorems for the solution of a third-order nonlinear differential equation in the vicinity of moving singular points in the complex domain, as well as constructs an analytical approximate solution, and obtains a priori estimates of the error of this approximate solution. The study was carried out using the modified method of majorants to solve this equation, which differs from the classical theory, in which this method is applied to the right-hand side of the equation The final point of the article is to conduct a numerical experiment to test the theoretical positions obtained.
\end{abstract}

\section{Introduction}

In the 19th century, Lazal Fuchs introduced the concept of a movable singular point. This feature is called movable due to the change in its location when the initial conditions change. The presence of such features automatically makes the classical theory unusable. Therefore, in the study of nonlinear differential equations, the first task is to prove the existence and uniqueness of the solution theorem.

Nonlinear differential equations have a wide range of applications, which indicates a great relevance in their application. In publication [1] wave processes in a rod are considered on the basis of the generalized Korteweg-de Fries-Burgers equation

$$
\frac{\partial u}{\partial t}-\frac{\partial \varphi(u)}{\partial x}=\mu \frac{\partial^{2} u}{\partial x^{2}}+m \frac{\partial^{3} u}{\partial x^{3}}, \quad m, \mu=\text { const } .
$$

In the stationary case and passing to specific values of the parameters, we will have the considered class of equations. In the publication [1], when considering the general form of the equation, the possible presence of movable singularities was not taken into account, which is a sufficient condition for its insolvability in quadratures. This fact actualizes the development of an approximate analytical method for solving such equations.

\footnotetext{
* Corresponding author: OrlovVN@mgsu.ru

$\uparrow$ Corresponding author: GasanovMV@mgsu.ru
} 
The next article [2] also investigates the wave processes arising in elastic beams, based on a nonlinear third-order differential equation. Similarly, as in the case of work [1], the specificity of nonlinearity and the presence of mobile singularities were not taken into account.

If the works [3-5] give a theoretical justification for taking into account the features of the applied class of nonlinear differential equations of the third order for the study of wave processes in elastic beams, then in the articles [6-9] the development of general theoretical provisions in the study of nonlinear differential equations with mobile singularities is given. Let us note a number of recent works with the application of this category of equations for building structures [10-16].

\section{Methods}

\subsection{Formulation of the problems}

Let's consider a nonlinear differential equation

$$
y^{\prime \prime \prime}=a_{7}(z) y^{7}+a_{6}(z) y^{6}+a_{5}(z) y^{5}+a_{4}(z) y^{4}+a_{3}(z) y^{3}+a_{2}(z) y^{2}+a_{1}(z) y+a_{0}(z)
$$

where $a_{i}(z)$ are holomorphic functions of a complex variable in some area.

Let us reduce equation (1) to normal form using the replacement indicated earlier [4] Let's consider the Cauchy problem

$$
\begin{gathered}
y^{\prime \prime \prime}=y^{7}+r(z), \\
\left\{\begin{array}{l}
y\left(z_{0}\right)=y_{0} \\
y^{\prime}\left(z_{0}\right)=y_{1} \\
y^{\prime \prime}\left(z_{0}\right)=y_{2}
\end{array}\right.
\end{gathered}
$$

\section{Theorem 1.}

1. Let $z^{*}$ be movable singular point of the solution of the Cauchy problem (2)-(3)

2. $r(x) \in C^{\prime}$ in area $\left|z^{*}-z\right|<\rho_{1}$ where $0<\rho_{1}=$ const;

3. $\exists M_{n}: \frac{\left|r^{(n)}\left(z^{*}\right)\right|}{n !} \leq M_{n} \quad M_{n}=$ const, then solution (4) - (5) is a meromorphic function

$$
y(z)=\left(z^{*}-z\right)^{-\frac{1}{2}} \sum_{0}^{\infty} C_{n}\left(z^{*}-z\right)^{n}
$$

In the area $\left|z^{*}-z\right|<\rho_{2}$, where $\rho_{2}=\min \left\{\rho_{1}, \frac{1}{(M+1)^{4}}\right\}, M=\sup _{n}\left\{\frac{\left|r^{(n)}\left(z^{*}\right)\right|}{n !}\right\}, n=0,1,2, \ldots$

\section{Proof.}

Representing the solution of eq. (4) in the vicinity of a moving singular point in the form of a meromorphic function

$$
y(z)=\left(z^{*}-z\right)^{\rho} \sum_{0}^{\infty} C_{n}\left(z^{*}-z\right)^{n}, C_{0} \neq 0
$$

By the hypothesis of the theorem $r(x)$ can be represented as

$$
r(z)=\sum_{0}^{\infty} A_{n}\left(z^{*}-z\right)^{n}
$$

Let's substitute (5) and (6) into equation (2):

$$
\sum_{0}^{\infty} C_{n}\left(z^{*}-z\right)^{n+\rho=3}(n+\rho)(n+\rho-1)(n+p-2)=\left(z^{*}-z\right)^{7 \rho} \sum_{0}^{\infty} C^{* * * *}{ }_{n}\left(z^{*}-z\right)^{n}+\sum_{0}^{\infty} A_{n}\left(z^{*}-z\right)^{n},
$$


where $C_{n}^{* * * *}=\sum_{i=0}^{n} C_{i} C_{j}^{* * *}, C_{n}^{* * *}=\sum_{i=0}^{n} C_{i}^{*} C_{j}^{* *}, C_{n}^{* *}=\sum_{i=0}^{n} C_{i}^{*} C_{j}^{*}, C_{n}^{*}=\sum_{i=0}^{n} C_{i} C_{n-i}$.

The last relation implies the need to fulfill the following conditions:

1) $n+\rho-3=n+7 \rho$;

2) $-(n-1)(n-3)(n-5) C_{n}=8\left(C_{n}^{* * * *+}+A_{\frac{n-7}{2}}\right)$, where $n=2 k+1, k=3,4, \ldots$;

3) $-(n-1)(n-3)(n-5) C_{n}=8 C_{n}^{* * * * *}$, where $n=2 k, k=0,1, \ldots$ and where $n=1,3,5$.

From the first equality it follows that $\rho=-\frac{1}{2}$. The second and third equalities are recurrence relations, which can be used to uniquely determine all the coefficients :

$$
C_{0}= \pm \sqrt[6]{\frac{15}{8}}, C_{1}=C_{2}=C_{3}=C_{4}=C_{5}=C_{6}=0, C_{7}=-\frac{8 A_{0}}{153}, C_{8}=0, C_{9}=-\frac{8 A_{1}}{297}, \ldots .
$$

Due to the uniqueness of the coefficients $C_{n}$ the uniqueness of the formal solution follows. Coefficient expressions $C_{n}$ were obtained by using the Maple mathematical package. Analysis of the expression of coefficients suggests a scoring structure for odds :

$$
\begin{aligned}
& \left|C_{3 k}\right| \leq \frac{8 M(M+1)^{6 k}}{(3 k-1)(3 k-3)(3 k-5)}=E_{3 k} ; \\
& \left|C_{3 k+1}\right| \leq \frac{8 M(M+1)^{6 k}}{3 k(3 k-2)(3 k-4)}=E_{3 k+1} ; \\
& \left|C_{3 k+2}\right| \leq \frac{8 M(M+1)^{6 k}}{(3 k+1)(3 k-1)(3 k-3)}=E_{3 k+2},
\end{aligned}
$$

where

$$
M=\sup _{n}\left\{\frac{\left|r^{(n)}\left(z^{*}\right)\right|}{n !}\right\}, n=0,1,2, \ldots
$$

Let us prove the validity of estimates (8). We restrict ourselves to the case for the coefficient $C_{3 k+3}$. Let $k=2 q+1, q=0,1,2 \ldots$. Then taking into account (7) and (8) we have:

$$
\begin{aligned}
& \left|C_{3 k+3}\right|=\left|\frac{8}{(3 k+2) \cdot 3 k \cdot(3 k-2)}\left(C_{3 k+3}^{* * * *}+A_{\frac{3 k-4}{2}}\right)\right|= \\
& =\left|\frac{1}{(3 k+2) \cdot 3 k \cdot(3 k-2)}\left(\sum_{i=0}^{k} C_{i}\left(\sum_{j=0}^{k-i} C_{j}^{*}\left(\sum_{l=0}^{k-i-j} C_{l}^{*}\left(\sum_{m}^{k-i-j-m} C_{m} C_{k-i-j-l-m}\right)\right)\right)+A_{k_{1}}\right)\right| \leq \\
& \leq \frac{8}{(3 k+2) \cdot 3 k \cdot(3 k-2)}\left(\sum _ { i - 0 } ^ { k } \frac { M ( M + 1 ) ^ { 6 i } } { ( 3 i + 2 ) \cdot 3 i ^ { * } \cdot ( 3 i - 2 ) } \left(\sum_{j-0}^{k-i} \frac{M(M+1)^{6 j}}{(3 j+2) \cdot 3 j^{*} \cdot(3 j-2)} \times\right.\right. \\
& \times\left(\sum _ { l - 0 } ^ { k - i - j } \frac { M ( M + 1 ) ^ { 6 l } } { ( 3 l + 2 ) \cdot 3 l ^ { * } \cdot ( 3 l - 2 ) } \left(\sum_{m-0}^{k-i-j-l} \frac{M(M+1)^{6 m}}{(3 m+2) \cdot 3 m^{*} \cdot(3 m-2)} \times\right.\right. \\
& \left.\left.\left.\left.\times \frac{M(M+1)^{6(k-i-j-l-m)}}{(3(k-i-j-l-m)+2) \cdot 3(k-i-j-l-m)^{*} \cdot(3(k-i-j-l-m)-2)}\right)\right)\right)+M\right) \leq \\
& \leq \frac{8 M^{5}(M+1)^{6 k}}{(3 k+2) \cdot 3 k \cdot(3 k-2)}\left(\sum _ { i - 0 } ^ { k } \frac { 1 } { ( 3 i + 2 ) \cdot 3 i ^ { * } \cdot ( 3 i - 2 ) } \left(\sum_{j-0}^{k-i} \frac{1}{(3 j+2) \cdot 3 j^{*} \cdot(3 j-2)} \times\right.\right.
\end{aligned}
$$




$$
\begin{aligned}
& \times\left(\sum _ { l - 0 } ^ { k - i - j } \frac { 1 } { ( 3 l + 2 ) \cdot 3 l ^ { * } \cdot ( 3 l - 2 ) } \left(\sum_{m-0}^{k-i-j-l} \frac{1}{(3 m+2) \cdot 3 m^{*} \cdot(3 m-2)} \times\right.\right. \\
& \left.\left.\left.\left.\times \frac{1}{(3(k-i-j-l-m)+2) \cdot 3(k-i-j-l-m)^{*} \cdot(3(k-i-j-l-m)-2)}\right)\right)\right)+M\right) \leq \\
& \leq \frac{8 M^{5}(M+1)^{6 k}+M}{(3 k+2) \cdot 3 k \cdot(3 k-2)} \leq \frac{8 M(M+1)^{6 k+6}}{(3 k+2) \cdot 3 k \cdot(3 k-2)},
\end{aligned}
$$

where

$$
\begin{gathered}
i^{*}=\left\{\begin{array}{l}
1, \text { if } i=0 \\
i, \text { if } i \neq 0
\end{array}, j^{*}=\left\{\begin{array}{l}
1, \text { if } j=0 \\
j, \text { if } j \neq 0
\end{array}, l^{*}=\left\{\begin{array}{l}
1, \text { if } l=0 \\
l, \text { if } l \neq 0
\end{array}, m^{*}=\left\{\begin{array}{c}
1, \text { if } m=0 \\
m, \text { if } m \neq 0
\end{array}\right.\right.\right.\right. \\
(k-i-j-l-m)^{*}=\left\{\begin{array}{c}
1, \text { if } m=k-i-j-l \\
(k-i-j-l-m), \text { if } m \neq k-i-j-l
\end{array}\right.
\end{gathered}
$$

In a similar way, we verify the validity of the estimates for the remaining cases $C_{3 k+1}$ and $C_{3 k+2}$.

Let's consider the series

$$
\sum_{1}^{\infty} E_{n}\left(z^{*}-z\right)^{\frac{n-1}{2}}
$$

which by virtue of (8) is majorizing for the series

$$
\sum_{1}^{\infty} C_{n}\left(z^{*}-z\right)^{\frac{n-1}{2}} \text {. }
$$

Due to the regularity of the coefficients of series (9) and (10), we represent series (9) in the form:

$$
\sum_{1}^{\infty} E_{n}\left(z^{*}-z\right)^{\frac{n-1}{2}}=\sum_{1}^{\infty} E_{3 k}\left(z^{*}-z\right)^{\frac{3 k-1}{2}}+\sum_{1}^{\infty} E_{3 k+1}\left(z^{*}-z\right)^{\frac{3 k}{2}}+\sum_{1}^{\infty} E_{3 k+2}\left(z^{*}-z\right)^{\frac{3 k+1}{2}} .
$$

For each series on the right-hand side of the last equality, taking into account estimates (8), according to the d'Alembert criterion, we have the convergence region

$$
\left|z^{*}-z\right|<\left(\frac{1}{(M+1)^{6}}\right)^{\frac{2}{3}}=\frac{1}{(M+1)^{4}} .
$$

Thus, we obtain the region of convergence of the correct part of series (9)

$$
\left|z^{*}-z\right|<\rho_{2},
$$

where $\rho_{2}=\min \left\{\rho_{1}, \frac{1}{(M+1)^{4}}\right\}$.

Theorem 1 proved allows constructing an analytical approximate solution in the form

$$
y_{N}(z)=\left(z^{*}-z\right)^{-\frac{1}{2}} \sum_{0}^{N} C_{n}\left(z^{*}-z\right)^{\frac{n}{2}}
$$

and getting its a priori estimate.

Theorem 2. Let points 2 and 3 of Theorem 1 hold and $z^{*}$ be a moving singular point of the solution of the Cauchy problem (2) - (3), then for the analytical approximate solution (11) in the domain

$$
\left|z^{*}-z\right|<\rho_{2}
$$

the error estimate is valid

$$
\Delta y_{N}(z)=\Delta,
$$

where 


$$
\begin{array}{r}
\Delta \leq \frac{8 M(M+1)^{2(N+!)}}{1-(M+1)^{6}\left|z^{*}-z\right|^{\frac{3}{2}}}\left|z^{*}-z\right|^{\frac{N}{2}}\left(\frac{1}{N(N-2)(N-4)}+\right. \\
\left.\quad+\frac{\left|z^{*}-z\right|^{\frac{1}{2}}}{(N+1)(N-1)(N-3)}+\frac{\left|z^{*}-z\right|}{(N+2) N(N-2)}\right)
\end{array}
$$

when $N+1=3 k$,

$$
\begin{aligned}
& \Delta \leq \frac{8 M(M+1)^{2 N}}{1-(M+1)^{6}\left|z^{*}-z\right|^{\frac{3}{2}}}\left|z^{*}-z\right|^{\frac{N}{2}}\left(\frac{1}{N(N-2)(N-4)}+\right. \\
& \left.+\frac{\left|z^{*}-z\right|^{\frac{1}{2}}}{(N+1)(N-1)(N-3)}+\frac{\left|z^{*}-z\right|}{(N+2) N(N-2)}\right)
\end{aligned}
$$

for option $N+1=3 k+1$, and

$$
\begin{aligned}
& \Delta \leq \frac{8 M(M+1)^{2(N-1)}}{1-(M+1)^{6}\left|z^{*}-z\right|^{\frac{3}{2}}}\left|z^{*}-z\right|^{\frac{N}{2}}\left(\frac{1}{N(N-2)(N-4)}+\right. \\
& \left.+\frac{\left|z^{*}-z\right|^{\frac{1}{2}}}{(N+1)(N-1)(N-3)}+\frac{\left|z^{*}-z\right|}{(N+2) N(N-2)}\right)
\end{aligned}
$$

for $N+1=3 k+2$, where $\rho_{2}=\min \left\{\rho_{1}, \frac{1}{(M+1)^{4}}\right\}, M=\sup _{n}\left\{\frac{\left|r^{(n)}\left(z^{*}\right)\right|}{n !}\right\}, n=0,1,2, \ldots$

Proof. Let us prove the theorem taking into account the case $N+1=3 k$. Let's write $\Delta y_{N}(x)$ :

$$
\Delta y_{N}(z)=\left|y(z)-y_{N}(z)\right|=\left|\sum_{0}^{\infty} C_{n}\left(z^{*}-z\right)^{\frac{n-1}{2}}-\sum_{0}^{N} C_{n}\left(z^{*}-z\right)^{\frac{n-1}{2}}\right|=\left|\sum_{N+1}^{\infty} C_{n}\left(z^{*}-z\right)^{\frac{n-1}{2}}\right|
$$

Taking into account the regularity in the estimates of the coefficients $C_{n}$, from theorem 1 , we obtain:

$$
\begin{gathered}
\Delta y_{N}(z)=\left|\sum_{N+1}^{\infty} C_{n}\left(z^{*}-z\right)^{\frac{n-1}{2}}\right| \leq \sum_{N+1}^{\infty}\left|C_{n}\right| \cdot\left|z^{*}-z\right|^{\frac{n-1}{2}} \leq \sum_{N+1}^{\infty} E_{3 k}\left|z^{*}-z\right|^{\frac{3 k-1}{2}}+\sum_{N+1}^{\infty} E_{3 k+1}\left|z^{*}-z\right|^{\frac{3 k}{2}}+ \\
+\sum_{N+1}^{\infty} E_{3 k+2}\left|z^{*}-z\right|^{\frac{3 k+1}{2}}=\sum_{N+1}^{\infty} \frac{8 M(M+1)^{6 k}}{(3 k-1)(3 k-3)(3 k-5)}\left|z^{*}-z\right|^{\frac{3 k-1}{2}}+ \\
+\sum_{N+1}^{\infty} \frac{8 M(M+1)^{6 k}}{3 k(3 k-2)(3 k-4)}\left|z^{*}-z\right|^{\frac{3 k}{2}}+\sum_{N+1}^{\infty} \frac{8 M(M+1)^{6 k}}{(3 k+1)(3 k-1)(3 k-3)}\left|z^{*}-z\right|^{\frac{3 k+1}{2}} \leq \\
\leq \frac{8 M(M+1)^{6 k}}{1-(M+1)^{6}\left|z^{*}-z\right|^{\frac{3}{2}}}\left|z^{*}-z\right|^{\frac{3 k-1}{2}}\left(\frac{\mid 1}{(3 k-1)(3 k-3)(3 k-5)}+\right. \\
\left.+\frac{\left|z^{*}-z\right|^{\frac{1}{2}}}{3 k(3 k-2)(3 k-4)}+\frac{\left|z^{*}-z\right|}{(3 k+1)(3 k-1)(3 k-3)}\right)= \\
=\frac{8 M(M+1)^{2(N+!)}}{1-(M+1)^{6}\left|z^{*}-z\right|^{\frac{3}{2}}}\left|z^{*}-z\right|^{\frac{N}{2}}\left(\frac{1}{N(N-2)(N-4)}+\right.
\end{gathered}
$$




$$
\left.+\frac{\left|z^{*}-z\right|^{\frac{1}{2}}}{(N+1)(N-1)(N-3)}+\frac{\left|z^{*}-z\right|}{(N+2) N(N-2)}\right)
$$

Similarly, we obtain expressions for $\Delta$ in the cases $N+1=3 k+1, N+1=3 k+2$. The estimates obtained are valid in the domain (12) in accordance with the conditions of the theorem.

\subsection{Numerical experiment}

Let's consider the Cachy problems (2) - (3), where

$r(z)=0 y(0)=1 / 4, y^{\prime}(0)=i, y^{\prime \prime}(0)=1, z^{*}=2.652717$. The calculation results for the Cauchy problem (2) - (3) are presented in Table 1.

Numerical characteristics of an analytically approximate solution. Table $\mathbf{1}$.

\begin{tabular}{|r|l|r|c|}
\hline$z_{2}$ & $y_{11}\left(z_{2}\right)$ & $\Delta_{1}$ & $\Delta_{2}$ \\
\hline 2.6523 & $78.3005+12.2448 i$ & 0.005 & 0.0001 \\
\hline
\end{tabular}

where $y_{11}\left(z_{2}\right)$ - analytically approximate solution (13); $\Delta_{1}-$ a priori error estimate; $\Delta_{2}-$ posterior evaluation. For $\Delta_{2}=0.005$ by Theorem 2 we define $N=15$. The terms from 12 to 15 in total do not exceed the required accuracy $-\varepsilon=0.0001$, therefore, at $N=11$ we get the value $y_{11}\left(z_{2}\right)$ with precision $\varepsilon=0.0001$.

\section{Conclusion}

In this article, we formulate and prove a theorem on the existence and uniqueness of the solution of one class of nonlinear differential equations of the third order in the vicinity of a moving singular point in a complex domain. The structure of the analytical approximate solution and an a priori estimate of the error are obtained. The results of a numerical experiment are presented, which confirm the theoretical results. A variant of optimizing a priori estimates using a posteriori is illustrated.

\section{References}

1. A.P. Chugainova // Nonstationary solutions of the generalized Korteweg - de Fries Burgers equation //// Proceedings of the Steklov Institute of Mathematics, 2013, 281, 204-212 DOI: https://doi.org/10.1134/S0371968513020179

2. Feng Yuqiang // Existence and uniqueness results for a third-order implicit differential equation, Computers and Mathematics with Applications 56 (2008) 2507-2514

3. Orlov V. N. , Gasanov M. V.//Study of wave processes in elastic beams and nonlinear differential equations with moving singular points// IOP Conference Series: Materials Science and Engineering 2020, doi:10.1088/1757-899X/1030/1/012081

4. Orlov V. N., Gasanov M. V // Existence theorem for a solution of one class of nonlinear differential equations of the third order with a polynomial right-hand side of the seventh degree in a neighborhood of a moving singular point// Vestnik ChGPU im. I. Ya. Yakovleva Series: Limit State Mechanics 2020. № 1 (43). C. 92-99 DOI: 10.37972/chgpu.2020.43.1.011 18. 
5. Aleroev T. S., Gasanov M. V // Perturbation of a moving singular point and an analytical approximate solution for a class of nonlinear third-order differential equations // Vestnik ChGPU im. I. Ya. Yakovleva Series: Limit State Mechanics. 2021. № 1 (47). C. 16-27.

6. Orlov V. N. // Method of approximate solution of the first, second differential equations of Painlevé and Abel. M.: MPGU, 2013. 174 c.

7. Orlov V. N. // Investigation of the approximate solution of the Abel differential equation in the vicinity of a moving singular point. // Vestnik MGTU im. N.E.Bauman. Series: Natural Sciences.2009. № 4 (35). C. 23-32.

8. Orlov V. N., Leontyeva T.Yu.// On the expansion of the domain for the analytical approximate solution of one class of nonlinear differential equations of the second order in the complex domain.// Vestnik Samarskogo Gos. tech. university. Ser. Phys.mat. science, 2020. T 2doi:10.14498/vsgtu1727

9. Orlov V. N., Yves B.B.// Existence theorem for a solution of one class of nonlinear differential equations of the fourth order with polynomial right-hand side of the second degree in the vicinity of a moving singular point// Bulletin of the Bashkir University..2018. T. 23, №4. C. 980-986.

10. Orlov V. N. , Zheglova Y.G. // Mathematical modeling of building structures and nonlinear differential equations // International Journal of Modeling, Simulation, and Scientific Computing. Vol. 11, No. 3 (2020) 2050026 (7 pages) World Scientific Publishing Company doi: 10.1142/S1793962320500269

11. Orlov V. N., Kovalchuk O.A. // Research of one class of nonlinear differential equations of third order for mathematical modelling the complex structures // IOP Conference Series: Materials Science and Engineering, 365, 2018. doi:10.1088/1757$899 \mathrm{X} / 365 / 4 / 042045$

12. Orlov V. N., Kovalchuk O.A., Linnik E.P., Linnik I.I. // Research into a Class of ThirdOrder Nonlinear Differential Equations in the Domain of Analyticity// Vestn. Mosk. Gos. Tekh. Univ. im. N.E. Baumana, Estestv. Nauki [Herald of the Bauman Moscow State Tech. Univ., Nat. Sci.], 2018, no. 4, pp. 24-35 (in Russ.). doi: 10.18698/18123368-2018-4-24-35

13. Orlov V. N., Kovalchuk O.A .// Mathematical modeling of complex structures and nonlinear differential equations with movable points // IOP Conf. Series: Materials Science and Engineering 456 (2018) 012122 IOP Publishing. doi:10.1088/1757$899 \mathrm{X} / 456 / 1 / 012122$

14. Orlov V. N., Kovalchuk O.A. // Mathematical problems of reliability assurance the building constructions// E3S Web Conf.Volume 97, 03031, 2019. XXII International Scientific Conference - Construction the Formation of Living Environment\| (FORM2019).doi:https://doi.org/10.1051/e3sconf/20199703031

15. Orlov V. N., Kovalchuk O.A. // An analytical solution with a given accuracy for a nonlinear mathematical model of a console-type construction (Scopus)// 18 Modelling and Methods of Structural Analysis IOP Conf. Series: Journal of Physics: Conf. Series 1425 (2020) 012127 IOP Publishing. doi:10.1088/1742-6596/1425/1/012127

16. Orlov V.N., Chichurin A. // On the theory of constructing a numerical-analytical solution of a cantilever beam bend nonlinear differential equation of the first order (Scopus)// Modelling and Methods of Structural Analysis IOP Conf. Series: Journal of Physics: Conf. Series 1425 (2020) 012129 IOP Publishing. doi:10.1088/17426596/1425/1/012129 